\title{
Resonant effects in a voltage-activated channel gating
}

\author{
Ewa Gudowska-Nowak ${ }^{a}$, Bartłomiej Dybiec ${ }^{a}$ and Henrik Flyvbjerg ${ }^{b}$ \\ ${ }^{a}$ Marian Smoluchowski Institute of Physics, Jagellonian University, Reymonta 4, \\ 30-059 Kraków, Poland; \\ ${ }^{b}$ Materials Research Department, Ris $\varnothing$ National Laboratory, Frederiksborgvej 399, DK-4000 \\ Roskilde, Denmark
}

\begin{abstract}
The non-selective voltage activated cation channel from the human red cells, which is activated at depolarizing potentials, has been shown to exhibit counter-clockwise gating hysteresis. We have analyzed the phenomenon with the simplest possible phenomenological models by assuming $2 \times 2$ discrete states, i.e. two normal open/closed states with two different states of "gate tension." Rates of transitions between the two branches of the hysteresis curve have been modeled with single-barrier kinetics by introducing a real-valued "reaction coordinate" parameterizing the protein's conformational change. When described in terms of the effective potential with cyclic variations of the control parameter (an activating voltage), this model exhibits typical "resonant effects": synchronization, resonant activation and stochastic resonance. Occurrence of the phenomena is investigated by running the stochastic dynamics of the model and analyzing statistical properties of gating trajectories.
\end{abstract}

Keywords: Hysteresis, stochastic resonance, channel gating, synchronization, resonant activation, signal to noise ratio, spectral amplification, power spectrum

\section{INTRODUCTION}

Ion channels are proteins that allow selective conduction of ions across a biological membrane. The transitions between an open (conducting) and a closed (nonconducting) conformation of proteins forming the channel can be registered by measuring movement of ions across the cell membrane. The switching of ion channels between different states is brought about ${ }^{1,2}$ by energy derived from thermal fluctuations, the electrical field and (or) binding the ligands. In particular, for voltage-gated ion channels discussed in this paper, kinetics between conducting and non-conducting states is controlled by the trans-membrane potential. The sequence of open and closed states can be measured in individual channels by the patch clamp technique ${ }^{2}$ to record the ionic current flowing through the single channel. The resulting time series recording is a starting point for kinetic analysis and modeling. A common assumption for most of the models used in the study of single channel gating is that the protein can dwell only in a few discrete states and that the switching between these states is considered random with the transition probabilities depending only on the present state, and not on the previous history of the channel. ${ }^{3}$ However, existing electrophysiological literature ${ }^{4-9}$ points out existence of some degree of memory in the function of ion channels. The identification of short- and long-term memory in single channel recordings could be then an essential tool to discriminate between various models of channel kinetics and interpretation of the data.

In relation to the channel memory a set of intriguing data have been reported by Kaestner et al. who have studied $^{6}$ voltage-activated cation channel in the human red blood cell (RBC) membrane, demonstrating that the degree of activation at a given membrane potential depended strongly on the prehistory of the channel. The gating exhibited hysteretic-like behavior with the quasi steady-state deactivation and activation curves displaced by about $25 \mathrm{mV}$. The effect has been explained ${ }^{10}$ by evaluating a kinetic model for voltage-activated channel gating that leads to a delayed response to a cycling external field in a way similar to the change of magnetization

Further author information:

E.G-N.: E-mail: gudowska@th.if.uj.edu.pl, Telephone: +48 (12) 663 5567, Fax: +48 (12) 6334079

B.D.: E-mail: bartek@th.if.uj.edu.pl, Telephone: +48 (12) 663 5566, Fax: +48 (12) 6334079

H.F.: E-mail: henrik.flyvbjerg@risoe.dk, Telephone: +45 4677 4104, Fax: +45 46774109 
in ferromagnetic materials under the influence of an external magnetic field. Alike memory-phenomena have been also discovered in the ligand-gated NMDA receptor, ${ }^{11}$ in voltage-gated HERG-like channels ${ }^{12}$ and in the gating kinetics of the vanilloid receptor ${ }^{13}$ which is a thermo-sensitive non-selective cation channel activated by noxious heat. In order to explain hysteretic behavior observed in channel response to external stimuli some of the authors ${ }^{12}$ suggested the existence of an ultra-slow inactivation process that effectively introduces a lag in the feedback between voltage and gating. Alternatively, ${ }^{13}$ a periodic sensitization of the channel on repeated heat stimulation has been assumed.

Viewed as a nonlinear and time-delayed response of a system to the cyclical variation of a control parameter, hysteresis is a fairly common phenomenon observed in bistable dynamical systems. ${ }^{14,15}$ In particular, it has been argued ${ }^{16-18}$ that the hysteresis loop area can be a useful quantity for identifying critical values of field parameters responsible for the onset of resonance-like response of the system and therefore helpful in designing field parameters for an optimal control. In the series of articles, Mahato, Shenoy and Jayannavar ${ }^{15,17}$ performed studies aimed to understand connection between the stochastic synchronization and stochastic resonance (SR) in a bistable system exhibiting hysteretic response to the external saw-tooth-type driving. They have shown that the hysteresis loop defined in such a system is a good measure of synchronization of passages from one well to the other under the influence of a Gaussian white noise. Moreover, at a particular value of the noise intensity, the maximum of the signal-to-noise ratio (SNR) is achieved thus demonstrating appearance of the stochastic resonance, that in turn, can be correlated with the hysteresis loop area. These findings suggest that hysteretic voltage-dependent gating may be closely related to documented observation of stochastic resonance in biological channels. ${ }^{19}$

Since the discovery of the SR phenomenon more than 20 years ago, the idea of stochastic resonance has gained a lot of interest in diverse disciplines of science where its presence has been demonstrated repeatedly ${ }^{20}$ in versatile applications. At the subcellular level, existence of the SR has been also proven in the ion channels ${ }^{19}$ where the sensory transduction of the signal (ionic current) can be optimized by the presence of intrinsic ambient noise. The result has provoked a question whether the SR is a collective effect occurring in some finite assemblies of channels or, perhaps could be also realized in a single channel activity. Further theoretical studies have revealed ${ }^{21}$ that in a single Shaker potassium channel the SR phenomenon can occur if the parameters that describe its functionality are set within the regime where the channel is predominantly dwelled in the closed state.

In this contribution we discuss occurrence of resonant effects in a model double-well system that has been used to describe voltage-dependent gating kinetics in the RBC cation channel. ${ }^{10}$ Section II presents basics of the reaction pathway modeling for that case. The interplay of voltage driving and thermal fluctuations are studied in an overdamped limit and lead to hysteresis, stochastic resonance and resonant activation. All those phenomena can be registered in the analysis of the channel gating kinetics, as discussed in Section III. Section IV is devoted to a standard two-state approximation within the Kramers scenario. Summary and closing remarks are presented in Section V.

\section{BISTABILITY AND REACTION PATHWAY}

The mechanism of voltage-gated ion channels was introduced in an early work of Hodgkin and Huxley who suggested that Boltzmann statistics describes their probability of being open or closed, including the voltagedependence of this probability. ${ }^{1,2}$ For the purpose of modeling, we assume the ion channel in question has only two states, open and closed. We associate an internal energy $E_{\text {open }}, E_{\text {closed }}$ with each of the two states, and assume that Boltzmann statistics describes the probability for a channel to be open or closed, with transitions between the two states being thermally activated barrier crossings in an energy landscape that we do not detail. Then the probability that a channel is open $\left(p_{\text {open }}\right)$ or closed $\left(p_{\text {closed }}\right)$ is

$$
p_{\text {open },(\text { closed })}=\frac{e^{-E_{\text {open },(\text { closed })}}}{e^{-E_{\text {open }}}+e^{-E_{\text {closed }}}}=\frac{1}{1+e^{ \pm \Delta E}}
$$

where all energies are given in units of $k_{\mathrm{B}} T$. In the case of voltage-gated channels, the transition between the open and the closed state possibly involves movement of charges in the trans-membrane field, reorientation of 
local polarization and conformational variation of channel protein that results in the change of energy $\Delta E$ of the system. With $\Delta E$ as above, Eq. (1) may be written ${ }^{10}$

$$
p_{\text {open }}(V)=\frac{1}{1+e^{-\alpha(V-\bar{V})}} \equiv f(V),
$$

where $\alpha \bar{V}$ replaces $\left(E_{\text {closed }}-E_{\text {open }}\right) / k_{\mathrm{B}} T, \bar{V}$ is the voltage at which $p_{\text {open }}=p_{\text {closed }}=1 / 2$ and $\alpha V$ parameterizes all other contributions to $\Delta E$. Equation (2) may be derived by assuming Kramer's scenario for the gating kinetics. ${ }^{22}$ This approach offers a description in which the configurational change of the channel protein is modeled by a multi-component "reaction coordinate" $x$. Since the potential energy of the entire system (the gate, channel protein and membrane environment) is a function of hundreds of relevant coordinates of the system, $x$ can be understood as an effective variable similar to its definition in the Marcus theory of charge-transfer reactions. ${ }^{23}$ The open and closed states of a channel can then be described as $x \approx 1$ and $x \approx-1$, respectively, with a potential (activation) barrier separating these two regions on the $x$-axis, and $x$ being confined to the regions near -1 and 1 by the potential taking large values elsewhere. Opening and closing of a channel is then modeled by a process similar to an activated uni-molecular chemical reaction pictured by a kinetic scheme

$$
\text { open } \underset{k_{\mathrm{o}}(t)}{\stackrel{k_{\mathrm{c}}(t)}{\rightleftharpoons}} \text { closed. }
$$

In order to capture the configurational change of the protein quantitatively, we introduce a parameterization for the reaction coordinate $x$ for this change by assuming that an equilibrium probability for the system to be in a conformational state $x$ is given by

$$
p(x)=\sum_{\sigma= \pm 1} p(\sigma, x)=e^{-W_{\mathrm{eff}}(x ; V)},
$$

where $\sigma$ describes a binary variable $\sigma= \pm 1$ discriminating between open $(\sigma=1)$ and closed $(\sigma=-1)$ states of the channel. A corresponding effective potential (cf. Fig. 1) takes on the form ${ }^{10}$

$$
W_{\text {eff }}(x ; V)=W(x)-\log \left[\cosh \left(\frac{1}{2}\left(\Delta E_{\text {gate }}+\Delta E_{\text {int }} x\right)\right)\right],
$$

where $W(x)$ describes the protein's internal energy as a function of $x$

$$
W(x)=\Delta E_{\text {conf }}\left(\frac{1}{4} x\left(3-x^{2}\right)+\frac{3}{16 x_{\max }}\left(1-x^{2}\right)^{2}\right),
$$

with $\Delta E_{\text {conf }}$ being a measure of the difference in the internal energy associated with the change of protein's configuration favoring open (closed) states of the gate. The parameter $x_{\max }$ has been chosen as the value of the reaction coordinate $x$ at which the local maximum, the top of the barrier between $x= \pm 1$ states is located; $\Delta E_{\text {gate }}=-\alpha(V-\bar{V})$ is contribution to the internal energy discriminating between the open (closed) states of the gate and $\Delta E_{\text {int }}=-\alpha \Delta V$ describes interaction energy between the gate and surrounding protein. In the above formula $\Delta V$ stands for the maximum intensity of driving external voltage $V$ and $\bar{V}$ is an average value of $V$ for which stationary probability $p_{\text {open }}$ achieves value $1 / 2$.

We now additionally assume that the membrane potential felt by a channel is not exactly constant, but changes, albeit slowly so compared to the rates of the gate opening and closing

$$
V(t)=\bar{V}-\Delta V \cos \Omega t .
$$

For $V(t)-\bar{V}$ large positive, stability analysis shows that conformational state $x=1$ is favored over $x=-1$ with an energy difference $\alpha \Delta V=\left|\Delta E_{\text {int }}\right|$. Similarly, for $V(t)-\bar{V}$ large negative

$$
W_{\text {eff }}(x ; V)=W(x)+\frac{1}{2} \alpha(V-\bar{V}+\Delta V x)+\log 2
$$




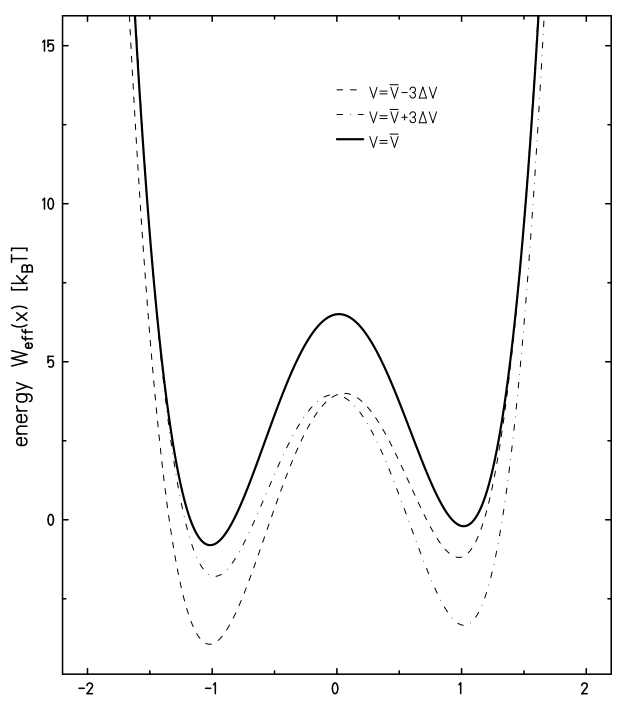

Figure 1. The effective potential for conformational changes, $W_{\text {eff }}(x ; V)$, for $V=\bar{V}, V=\bar{V}+3 \Delta V$ and $V=\bar{V}-3 \Delta V$. Parameters of the potential (in units of $k_{\mathrm{B}} T$ ) $\Delta E_{\text {conf }}=0.6, \Delta E_{\text {gate }}=9.72, \Delta E_{\text {int }}=2.16, x_{\max }=0.018, \Delta V=12$ and $\bar{V}=66$. The set of parameters has been $\operatorname{chosen}^{10}$ to model hysteretic behavior observed in opening probabilities in the RBC channel. ${ }^{6}$

and the barrier at $x_{\max }$ becomes lowered by $\frac{1}{2} \alpha|V-\bar{V}|=\frac{1}{2}\left|\Delta E_{\text {gate }}(V)\right|$ favoring state $x=-1$ over $x=1$. Note that for large differences $V(t)-\bar{V}$, addition of time-dependent driving does not change characteristic relaxation frequencies of the system within the wells of the potential $W_{\mathrm{eff}}(x)$. Direct evaluation of $\omega_{x_{\max }}^{2}=W_{\mathrm{eff}}^{\prime \prime}\left(x_{\max }\right)$ and $\omega_{ \pm 1}^{2}=W_{\text {eff }}^{\prime \prime}(x= \pm 1)$ yields $\omega_{x_{\max }}^{2} \approx-3 \Delta E_{c} / 4, \omega_{ \pm 1}^{2} \approx 3 \Delta E_{c} /\left(2 x_{\max }\right)$ for values of $x_{\max }$ close to 0 . We further assume that fluctuations of the collective variable $x$ quickly equilibrate, i.e. their dynamics can be well approximated by an overdamped Langevin equation

$$
\frac{d x}{d t}=-\frac{d W_{\mathrm{eff}}(x ; V)}{d x}+\xi(t)
$$

with $\xi(t)$ representing effects of thermal noise modeled by uncorrelated Gaussian white noise, i.e $\left\langle\xi(t) \xi\left(t^{\prime}\right)\right\rangle=$ $\sigma^{2} \delta\left(t-t^{\prime}\right)$. For convenience, we set the friction coefficient $\gamma=1$ that rescales the time $t$ in the above equation. Fig. 2 presents samples of stochastic trajectories $X(t)$ simulated from the above Langevin equation at constant intensity $\sigma^{2}=8$ of the additive noise (mimicking conditions of a constant temperature of the thermal medium). They model records of current in a biological channel switching between conducting and non-conducting conformations. As expected from the former analysis, at large positive values of the gating voltage $V$, the channel is predominantly open with current fluctuating due to the local conformational variations around the open state $(x=1)$. At $V=\bar{V}$ none of the states is favored, whereas for large negative $V$ a preferential stay in a closed state is visible. Increasing the noise intensity at a constant value of the voltage cycling frequency causes a trajectory to fluctuate wildly between both wells of the effective potential shortening the time the process spends in the vicinity of the metastable points $x= \pm 1$ and eventually, destroying the pattern of the signal switching between "open" and "closed" states.

From the residence time frequency histograms for the open and closed states, we have replotted probability of the channel being open as a function of the driving voltage. Fig. 3 presents estimated opening probabilities for various intensities of thermal noise.

By increasing the activating voltage from $V=\bar{V}$ to $V=\bar{V}+3 \Delta V$ and reversing the direction of voltage changes to $V=\bar{V}-3 \Delta V$, an apparent hysteretic shape of the $p_{\text {open }}(V)$ curves is observed (cf. Fig. 3). The hysteresis changes its shape and area with increasing intensity of the thermal noise and disappears for large values of $\sigma^{2}$. Through cycling $V$, the height of the potential barrier dividing wells of open and closed states is 

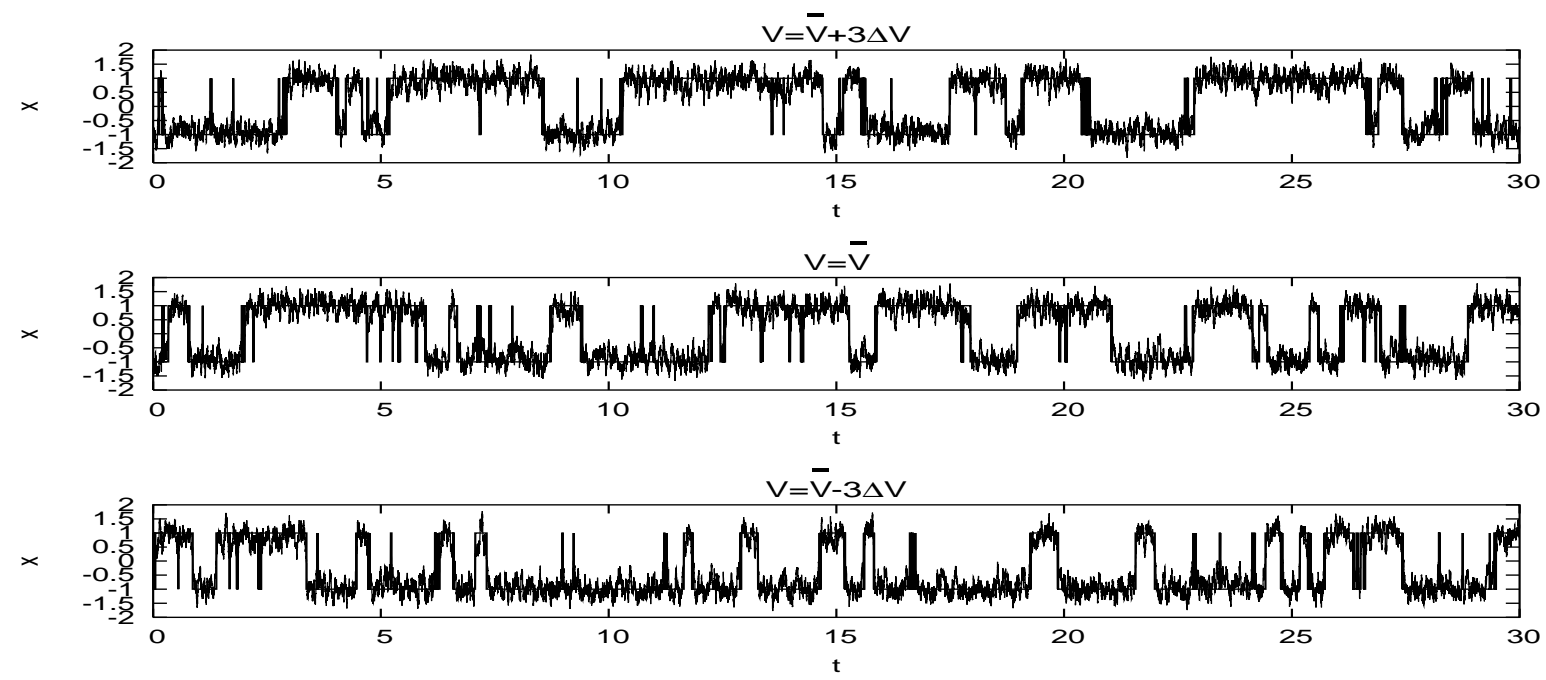

Figure 2. Sample realizations of the stochastic process $X(t)$, for the two state and the continuous model, derived from numerical simulations of Eq. (9). Time step of simulations has been set up to $10^{-3}$ with the frequency of the cycling voltage $\Omega=1$. Trajectories have been run for the duration time $T \approx 10 \pi$. Initial value of $X$ has been sampled from the uniform distribution on the interval $[-1.5,1.5]$.
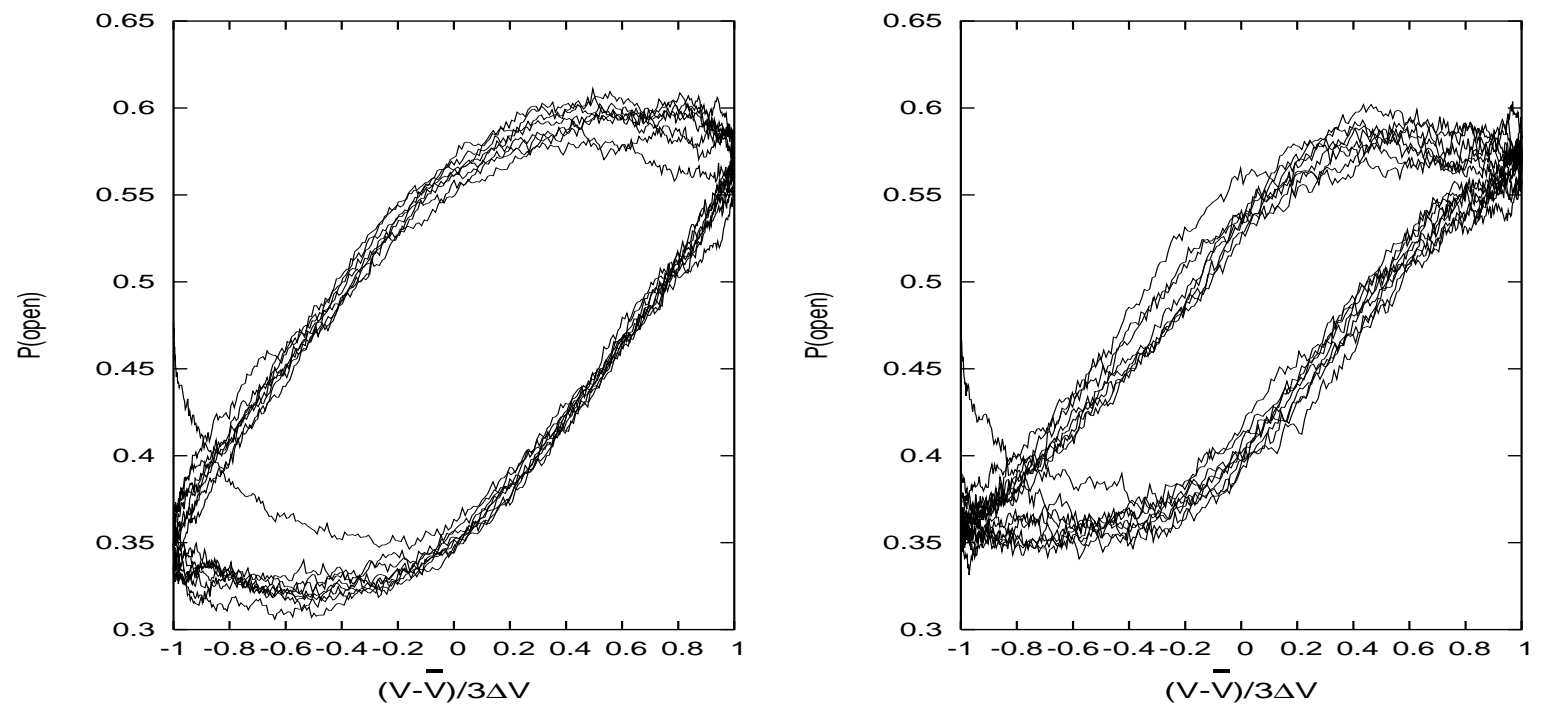

Figure 3. The open state probability $p_{\text {open }}$ as a function of the membrane potential $V$. Probability has been estimated from the frequency histograms for $3 \times 10^{3}$ trajectories mimicking passages between different conductance levels. Intensity of the thermal noise $\sigma^{2}=6$ (left panel) and $\sigma^{2}=8$ (right panel); time step of the simulation $d t=10^{-3}$. 

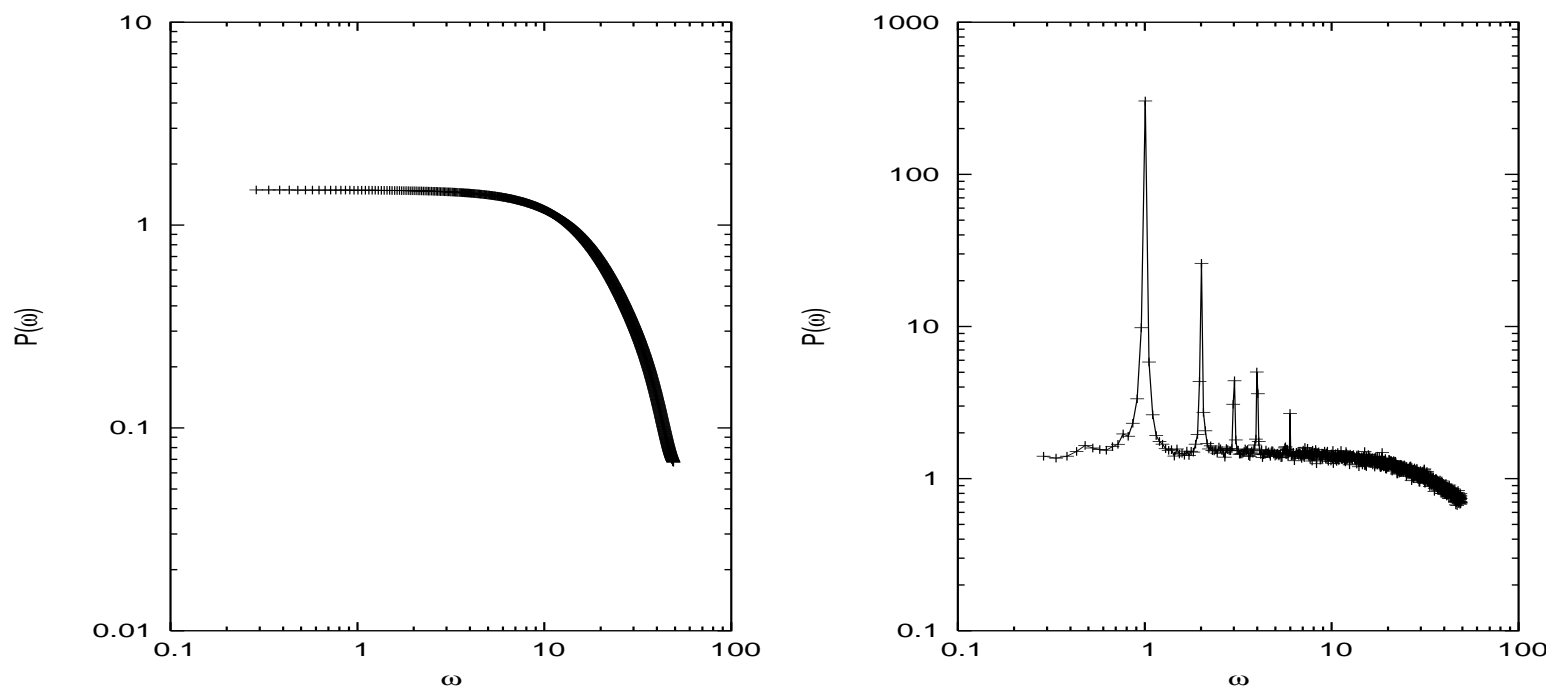

Figure 4. Power spectra of the channel gating process for the two-state (left panel) and continuous model (right panel), as simulated from Eq. (9) at the intensity of the additive thermal noise $\sigma^{2}=0.2$ and $\Omega=1$.

now itself a time-dependent variable. From other contexts, ${ }^{19,20,24,25}$ it is known that this presence of periodic driving force and additional noise (fluctuations) may lead to several nontrivial resonant effects which we are now going to address in the forthcoming section.

\section{DYNAMICAL HYSTERESIS, STOCHASTIC RESONANCE AND RESONANT ACTIVATION IN CHANNEL GATING KINETICS}

An essential property of the stochastic process $\{X(t)\}$ is interdependence of the random variable $X$ measured by the covariance function $C(t, \tau)=\langle X(t+\tau) X(t)\rangle$ which through the Wiener-Khinchin theorem is related to the power spectrum of the process $\{X(t)\}$. In the framework of Fourier transformation, properties of the covariance function may also be read off from its Fourier transform for a discretized version of trajectories $X(t)$. Figs. 4 and 5 present plots of the power spectral density as a function of frequency from a simulation of Eq. (9). The power spectra have been obtained from discrete periodograms for two different values of the intensity of the additive noise $\xi(t)$. At low noise intensity $\sigma^{2}=0.2$, the power spectrum exhibits sharp maxima at multiples of the unit frequency of the driving voltage $V$ (cf. right panel of Fig. 4). The existence of spikes in the power spectrum is a general feature for periodically driven systems ${ }^{26}$ and reflects (asymptotically) periodic character of the probability distribution $p(x, t)$. This feature is absent in the power spectrum of the two-state model (see left panel of Fig. 4). In this case any trajectory reaching the top of the potential barrier at $x_{\max }$ is assumed to switch the system from one state to another, so that the outgoing "signal" $X(t)$ is filtered and stored as a binary series of \pm 1 values. On increasing the noise intensity (or, equivalently temperature of the channel environment) to $\sigma^{2}=6$, the structure of multiple maxima washes out leaving the dominant peak at $\omega \approx \Omega=1$ observable for both - the two state approximation and the continuous model (cf. Fig. 5). For large values of $\omega$, power spectra scale to the $\omega^{-2}$ form reflecting exponentially decaying memory of the gating process $\{X(t)\}$.

The spectral amplification (an integrated power in the time-averaged power spectral density at $\omega \approx \Omega=1$ ) has been calculated (cf. Fig. 6) according to the interpretation due to Jung and Hänggi. ${ }^{26}$ The mean value of the $\{X(t)\}$ process has been obtained by averaging over the ensemble of various realizations of the additive noise. For long times $t \rightarrow \infty$ an asymptotic trajectory looses memory of the initial conditions and becomes a periodic function of time. Its square amplitude is then proportional to the spectral amplification expressed as the ratio of integrated power stored in the delta-like peaks of the power spectra at $\pm \Omega$ to a total power of the modulation signal in $V$. 


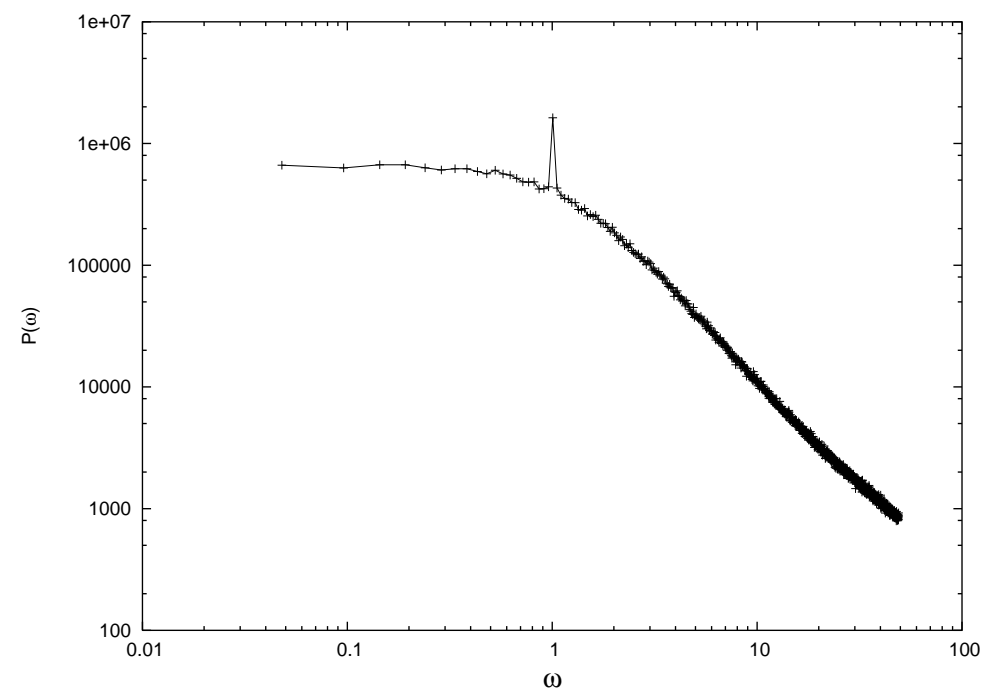

Figure 5. Power spectrum of the channel gating process for the continuous model simulated from Eq. (9). Intensity of the additive thermal noise $\sigma^{2}=6$ and $\Omega=1$.

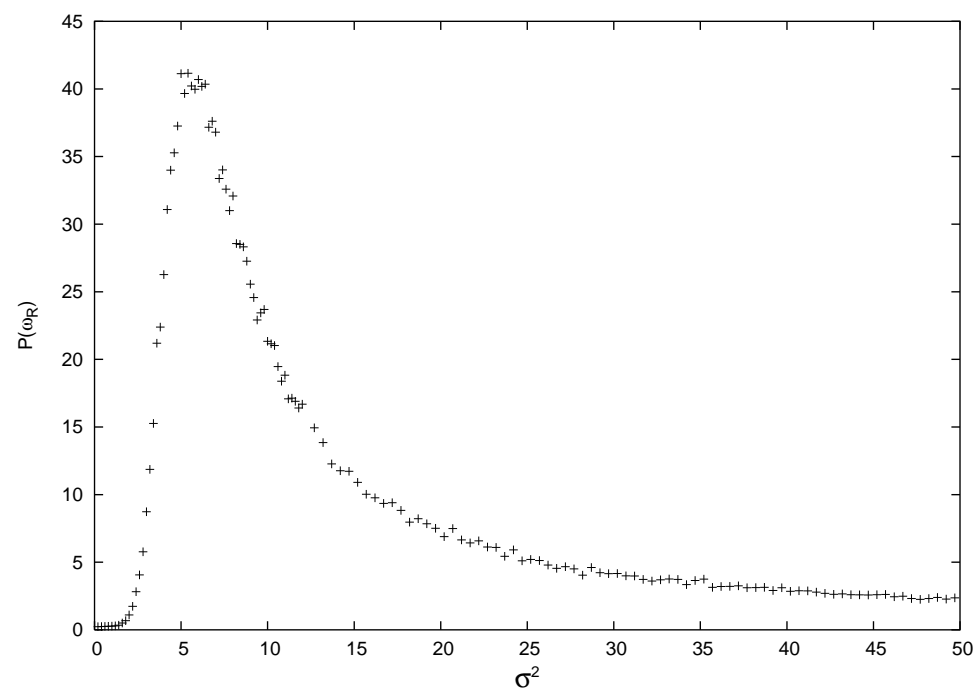

Figure 6. Spectral amplification, in arbitrary units for the continuous model, of the signal (registered as a trajectory passing from the left to the right well of the potential $\left.W_{\text {eff }}(x ; V)\right)$ for $\Omega=1$ at various noise intensities $\sigma^{2}$. 

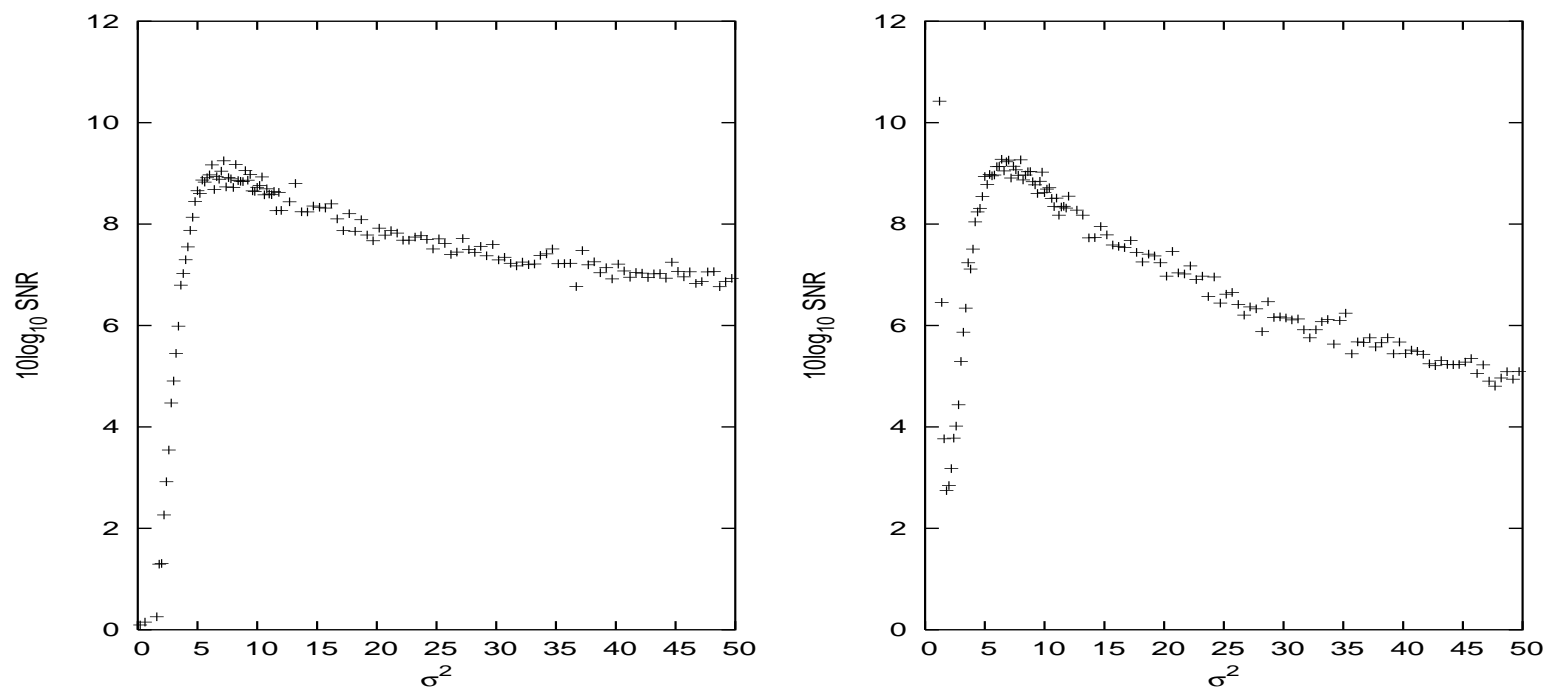

Figure 7. The signal-to-noise ratio for $\Omega=1$ for the two state model (left panel) and for the continuous model (right panel), as a function of the noise strength $\sigma^{2}$. The decaying part of the curve, in the right panel, represents relaxation processes in the potential well, details in the text.

Spectral amplification displays a typical bell-shaped stochastic resonance behavior as a function of increasing noise intensity with a characteristic peak observed for $\sigma^{2} \approx 6$ for both models under study. Similarly, evaluation of the signal-to-noise (SNR) ratio for the potential under study produces curve (Fig. 7) that exhibits nonmonotonic resonant behavior for $\sigma^{2} \approx 6$ and characteristic divergence for the noise strength $\sigma^{2} \rightarrow 0$. For small $\sigma^{2}\left(\sigma^{2}<2\right)$ barrier crossing events are rare events, and the decaying part of the SNR curve (cf. right panel of Fig. 7) corresponds to relaxation processes within the potential well. This feature is absent for the two state model approximation. In accordance with the former studies of Mahato and Jayannavar, ${ }^{15,17}$ the maximum area of the hysteresis curve is observed for the noise intensity at which the stochastic resonance is registered.

The same system may also exhibit a different resonant behavior - so called resonant activation (RA) 25,27 expressed by the shortest time of passage over a fluctuating potential barrier in the presence of an additive noise. As expected based on previous theoretical studies, ${ }^{25,27,28}$ the RA occurs typically in the bistable system under broad circumstances of varying shape of the potential barrier and qualities of fluctuations. In the case of the modeled channel system, the resonant activation is brought up by an interplay of cyclic (deterministic) variations of the barrier and an additive thermal noise (Fig. 8).

In order to examine the statistics of the first passage times FPT for various shapes of the potential $W_{\text {eff }}(x ; V)$ introduced by the cycling voltage $V(t)$ own program performing Monte Carlo simulations has been used. The trajectories Eq. (9) have been run starting from the left well until the top of the barrier has been hit, where by the assumption, the trajectory has been absorbed. A position of the top of the barrier $x_{\max }$ depends on the driving voltage (see Fig. 9) and for a given set of the parameters smoothly changes between $x_{\max } \approx 0.06$ and $x_{\max } \approx-0.03$. Within the error limits of the simulations the resonant activation becomes visible at the noise intensity $\sigma^{2}=2$ and the effect vanishes with increasing values of $\sigma^{2}$.

\section{TWO STATE APPROXIMATION}

In order to discuss the configurational change of the protein quantitatively we have introduced a parameterization of the reaction pathway for this change. We now explore the consequences of the specific form of $W_{\text {eff }}(x ; V)$, approximating the thermal barrier crossing with the simple Kramers scenario. In doing that, our description captures, albeit approximatively only, the consequences of a much larger set of potentials which are generic for studying bistability and SR phenomena. 


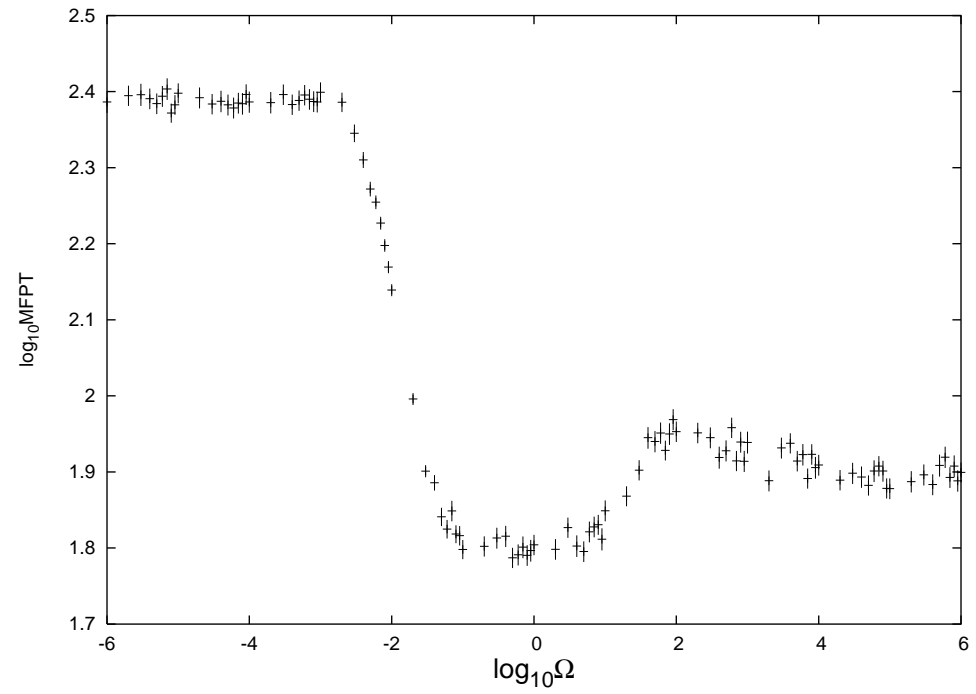

Figure 8. The MFPT for trajectories starting from the left minimum of the potential $W_{\text {eff }}(x ; V)$ as a function of driving voltage frequency $\Omega$ at the noise strength $\sigma^{2}=2$. Numerical results were obtained by use of Monte Carlo simulation of Eq. (9) with time step $d t=10^{-5}$ and averaged over $N=10^{3}$ realizations. Error bars represent deviation from the mean.

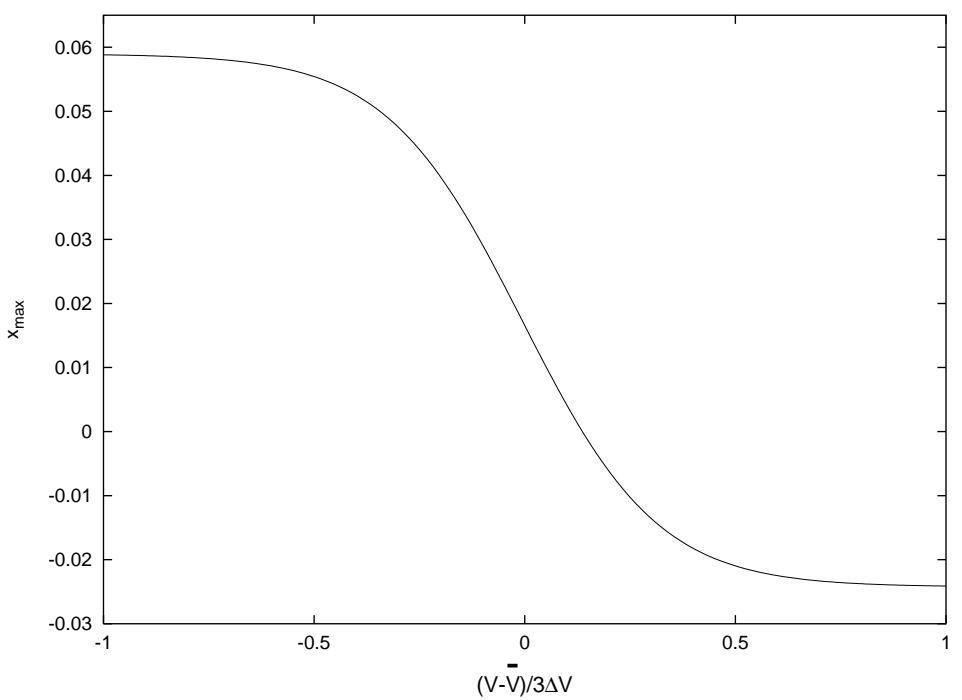

Figure 9. Location of the barrier top for periodically perturbed potential as a function of the driving voltage. Parameters $\Delta E_{\text {conf }}, \Delta E_{\text {int }}, \Delta E_{\text {gate }}, \Delta V$ and $\bar{V}$ as in Fig. 1 . 
In the overdamped Kramer's scenario, ${ }^{29}$ after assuming that the thermal energy $k_{\mathrm{B}} T$ is much smaller than the respective barrier height $\Delta W^{\dagger}=W_{\text {eff }}\left(x_{\max } ; V\right)-W_{\text {eff }}( \pm 1 ; V)$, the reaction coordinate undergoes a creeping motion and an adiabatic elimination of the velocity $\dot{x}$ leads to the Langevin equation (9). The time evolution of the corresponding probability $p(x, t)$ is governed by the Smoluchowski equation. The spatial-diffusion rate in the potential $W_{\text {eff }}(x ; V)$ can be then evaluated by a steepest-descent approximation from the quadrature formulas for the mean first passage time (MFPT)

$$
\begin{aligned}
k_{\mathrm{o}} & =k_{-1 \rightarrow+1}(V(t)) \\
& =\nu_{-} e^{-\left(W_{\mathrm{eff}}\left(x_{\max } ; V(t)\right)-W_{\mathrm{eff}}(-1 ; V(t))\right)}, \\
k_{\mathrm{c}} & =k_{+1 \rightarrow-1}(V(t)) \\
& =\nu_{+} e^{-\left(W_{\mathrm{eff}}\left(x_{\max } ; V(t)\right)-W_{\mathrm{eff}}(+1, V(t))\right)},
\end{aligned}
$$

where the subdominant factor $\nu_{ \pm}=\left(\omega_{ \pm 1} \omega_{x_{\max }}\right) / 2 \pi$ contains product of the characteristic angular frequency inside the metastable minimum $(x= \pm 1)$ and an angular frequency at the top of the potential barrier.

For a sufficiently high barrier separating open and closed states, the probability of $x$ taking values in between vanishes and we can work with the integrated probability of $x$ being near -1 or $1, n_{\text {closed }}(t)=\int_{-\infty}^{x_{\max }-\delta} p(x, t) d x$ and $n_{\text {open }}(t)=\int_{x_{\max }+\delta}^{\infty} p(x, t) d t$ where $\{-\delta, \delta\}$ describes an interval in the vicinity of the metastable $x_{\max }$. The integrated probabilities satisfy $n_{\text {closed }}(t)+n_{\text {open }}(t)=1$ and the linear kinetic equation

$$
\frac{d n_{\text {open }}(t)}{d t}=k_{\mathrm{o}} n_{\text {closed }}(t)-k_{\mathrm{c}} n_{\mathrm{open}}(t) .
$$

Under stationary conditions the ratio of the reaction rates can be related to the difference in the energy by the equilibrium constant

$$
K=\frac{k_{\mathrm{c}}}{k_{\mathrm{o}}}=\frac{n_{\text {closed }}}{n_{\text {open }}}=\frac{\nu_{+}}{\nu_{-}} e^{-\left[W_{\text {eff }}(-1 ; V(t))-W_{\text {eff }}(1 ; V(t))\right]} .
$$

Rearrangement of this equation gives a formula for the probability of the channel being open Eq. (2)

$$
p_{\text {open }}=\frac{1}{1+k_{\mathrm{c}} / k_{\mathrm{o}}}
$$

Thus $\Delta W_{\text {eff }}$ is related to the ratio of kinetic rates for the "forward" and "backward" reactions. Experimental estimates of the opening and closing rates can be found from the voltage-dependent dwell times determined from experimental recordings as inverse of averages i.e. $k_{\mathrm{o}}=\left\langle T_{\mathrm{c}}\right\rangle^{-1}, k_{\mathrm{c}}=\left\langle T_{\mathrm{o}}\right\rangle^{-1}$ where $\left\langle T_{\mathrm{c}, \mathrm{o}}\right\rangle$ are average dwell times in the closed (open) states. The gating dynamics described by Eq. (11) can be otherwise considered as a two-state Markovian process ${ }^{30-32}$ with a time-dependent transition probabilities between the states. A two state model of that type is known to describe a stochastic resonance ${ }^{20,33}$ and with both periodically and randomly modulated rates has been investigated.. ${ }^{32}$

The general solution of Eq. (11) can be readily found

$$
\begin{aligned}
n_{\text {open }}(t) & =e^{-\left[K_{\mathrm{c}}(t, 0)+K_{\mathrm{o}}(t, 0)\right]} n_{\mathrm{open}}(0) \\
& +C \int_{0}^{t} d s k_{\mathrm{o}}(s) e^{-\left[K_{\mathrm{c}}(t, s)+K_{\mathrm{o}}(t, s)\right]},
\end{aligned}
$$

where

$$
K_{\mathrm{o}, \mathrm{c}}(t, s) \equiv \int_{\mathrm{s}}^{t} d t^{\prime} k_{\mathrm{o}, \mathrm{c}}\left(t^{\prime}\right)
$$

and

$$
k_{\mathrm{o}, \mathrm{c}}=\kappa_{\mathrm{o}, \mathrm{c}} e^{-\alpha_{\mathrm{o}, \mathrm{c}} V}\left[1+\alpha_{\mathrm{o}, \mathrm{c}} \cos t+\lambda_{\mathrm{o}, \mathrm{c}} \eta(t)\right],
$$

where $\eta(t)$ stands for other sources of (nonthermal) noises of intensity $\lambda_{\circ, \mathrm{c}}$ that can perturb the transition rates. Note, that the Kramers rate formula is derived under the assumption that the probability density within the 
well is close to its equilibrium distribution, so that in order to use the modified Kramers rate with a periodic perturbation of the voltage, its frequency must be much slower than the characteristic rate for the probability to equilibrate within the well. Such an adiabatic approximation is valid if the second derivative of the effective potential $W_{\text {eff }}(x ; V)$ at $x= \pm 1$, i.e. $\omega_{ \pm 1}$ is much bigger than 1 (in our analysis the characteristic period of the cycling voltage has been set up to 1 ). Parametric plots of the cumulative open probability, as defined by $n_{\text {open }}$ in function of $V$ display then the dynamic hysteresis whose shape and loop-area depend on the ratio of the driving voltage frequency to the overall relaxation rate $k=k_{\mathrm{o}}+k_{\mathrm{c}}$. The hysteresis disappears only in case of driving that has time scale significantly different from the inverse of rates $k_{\mathrm{o}, \mathrm{c}}$, i.e. when it is either very slow or very fast. In such situations, the response of the system measured in terms of the integrated probabilities $n_{\text {open,closed }}$ depends on the applied voltage but not on its frequencies (slow driving), or follows (fast driving) average, time independent rates. In consequence, hysteretic behavior may be considered a generic for systems exhibiting the SR phenomenon. For the periodically driven two state process, recent analysis performed by Talkner ${ }^{34}$ has shown that studies of the entrance time densities in the system can detect the synchronization of the driving force and the driving process. However, a more detailed comparison of stochastic resonance and synchronization ${ }^{17,34}$ shows that the effects are closely related but not identical.

\section{SUMMARY}

There is no unique consensus in the SR literature on how to measure the SR effect. The most popular performance measures used in the study depend on the forcing signal and noise and can vary from system to system. The common approach to characterize SR is the SNR that measures how much the system output contains the input signal frequency $\Omega$. An adiabatic approximation ${ }^{20,33}$ can then give an explicit SNR ratio for the quadratic bistable potential. Alternative ways to measure SR are related to the cross-correlation measures and estimates of the density probability of residence time ${ }^{17}$ and escape rates. ${ }^{34}$

In this work we have concentrated on a class of resonant effects that can be exhibited in the gating kinetics of the voltage-driven biological channel. Our analysis reproduces all typical phenomena that are characteristic for the two-state system driven by fluctuations and periodic forcing. Albeit the latter does not function here as an additive periodic force but rather modulates the height of the free energy barrier that discriminates between different conformational states of the gate, its presence and interference with inherent thermal fluctuations leads to a fine-tuning of the gating kinetics exhibited by stochastic resonance and resonant activation.

\section{ACKNOWLEDGMENTS}

The Authors acknowledge the financial support from the Polish State Committee for Scientific Research (KBN) through grants 1P03B06626 and 2P03B08225.

\section{REFERENCES}

1. A. L. Hodgin, and A. F. Huxley, "A quantitative description of membrane current and its application to conduction and excitation in nerve," J. Physiol. (Lond.) 117, pp. 500-544, 1952.

2. B. Hille, Ionic Channels of Excitable Membranes, Sinauer Associates Inc, Sunderl, MA, 1992.

3. D. Colquhoun, and A. G. Hawkes, "On the stochastic properties of single ion channels," Proc. R. Soc. Lond. Ser. B. 211, pp. 205-235, 1981.

4. P. Labarca, J. A. Rice, D. R. Fredkin, and M. Montal, "Kinetic analysis of channel gating. Application to the cholinergic receptor channel and the chloride channel from Torpedo californica," Biophys. J. 47, pp. 469-478, 1985 .

5. M. B. Jackson, B. S. Wong, C. E. Morris, and H. Lecar, "Successive openings of the same acetylcholine receptor channel are correlated in open time," Biophys. J. 42, pp. 109-114, 1983.

6. L. Kaestner, P. Christophersen, I. Bernhard, P. Bennekou, "The non-selective voltage-activated cation channel in the human red blood cell membrane: reconciliation between two conflicting reports and further characterisation," Bioelectrochem. 52, pp. 117-125, 2000.

7. A. Fuliński, Z. Grzywna, I. Mellor, Z. Siwy, and P. N. R. Usherwood, "Non-Markovian character of ionic current fluctuations in membrane channels," Phys. Rev. E 58, pp. 919-924, 1998. 
8. Sz. Mercik, K. Weron, and Z. Siwy, "Statistical analysis of ionic current fluctuations in membrane channels," Phys. Rev. E 60, pp. 7343-7348, 1999.

9. W. A. Varanda, L. S. Liebovitch, J. N. Figueiroa, and R. A. Nogueira, "Hurst analysis applied to the study of single calcium-activated potassium channel kinetics," J. Theor. Biol. 206, pp. 343-353, 2000.

10. E. Gudowska-Nowak, P. Bennekou, P. Christophersen, and H. Flyvbjerg, "Modelling Hysteresis Observed in a Voltage-Activated Ion Channel," work in progress.

11. L. M. Nowak, and J. M. Wright, "Slow voltage-dependent changes in channel open-state probability underlie hysteresis of NMDA responses in $\mathrm{Mg}^{2+}$-free solutions," Neuron 8, pp. 181-187, 1992.

12. P. S. Pennefather, W. Zhou, and T. E. DeCoursey, "Idiosyncratic gating of HERG-like $\mathrm{K}^{+}$channels in microglia," J. Gen. Physiol. 111, pp. 795-805, 1998.

13. H. Xu, I. S. Ramsey, S.A. Kotecha, M. M. Moran, J. A. Chong, D. Lawson, P. Ge, J. Lilly, I. Silos-Santiago, Y. Xie, P. S. DiStefano, R. Curtis, and D. E. Clapham, "TRPV3 is a calcium-permeable temperature-sensitive cation channel," Nature 418, pp. 181-186, 2002.

14. P. Jung, G. Gray, R. Roy, and P. M, andel, "Scaling law for dynamical hysteresis," Phys. Rev. Lett. 65, pp. 1873-1876, 1990.

15. M. C. Mahato, and A. M. Jayannavar, "Some stochastic phenomena in a driven double-well system," Physica A 248, pp. 138-154, 1998.

16. E. C. Gage, and L. M, andel, "Hysteresis effects in the two-mode dye ring laser," J. Opt. Soc. Am. B 6, pp. 287-291, 1989.

17. M. C. Mahato, and A. M. Jayannavar, "Relation between stochastic resonance and synchronization of passages in a double-well system ," Phys. Rev. E 55, pp. 6266-6269, 1997.

18. J. C. Phillips, and K. Schulten, "Diffusive hysteresis at high and low driving frequencies," Phys. Rev. E 52, pp. 2473-2477, 1995.

19. S. M. Bezrukov, and I. Vodyanoy, "Stochastic resonance in non-dynamical systems without response thresholds," Nature 385, pp. 319-321, 1997.

20. L. Gammaitoni, P. Hänggi, P. Jung, and F. Marchesoni, "Stochastic resonance," Rev. Mod. Phys. 70, pp. 223-287, 1998.

21. I. Goychuk, P. Hänggi, "Stochastic resonance in ion channels characterized by information theory," Phys. Rev. E 61, pp. 4272-4280, 2000.

22. D. Sigg, H. Qiau, and F. Bezanilla, "Kramers' Diffusion Theory Applied to Gating Kinetics of VoltageDependent Ion Channels," Biophys. J. 76, pp. 782-803, 1999.

23. A. M. Kuznetsov, and J. Ulstrup, Electron Transfer in Chemistry, and Biology. An Introduction to the Theory, Wiley, Chichester, 1999.

24. A. Fuliński, "Noisy chemical equilibria and flow reactor's yield," J. Chem. Phys. 114, pp. 3109-3119, 2001.

25. J. Iwaniszewski, I. K. Kaufman, P. V. E. McClintock and A. J. McKane, "Resonances while surmounting a fluctuating barrier," Phys. Rev. E 61, pp. 1170-1175, 2000.

26. P. Jung, and P. Hänggi, "Amplification of small signals via stochastic resonance," Phys. Rev. A 44, pp. 8032-8042, 1991.

27. A. Zürcher, and Ch. R. Doering, "Thermally activated escape over fluctuating barriers," Phys. Rev. E 47, pp. 3862-3869, 1993.

28. B. Dybiec, and E. Gudowska-Nowak, "Influence of the barrier shape on resonant activation," Phys. Rev. E 66, pp. 026123-1-6, 2002.

29. P. Hänggi, P. Talkner, and M. Borkovec, "Reaction-rate theory: fifty years after Kramers," Rev. Mod. Phys. 62, pp. 251-341, 1990.

30. A. Fuliński, "Relaxation, noise-induced transitions, and stochastic resonance driven by non-Markovian dichotomic noise," Phys. Rev. E 52, pp. 4523-4526, 1995.

31. P. Talkner, "Markov processes driven by quasi-periodic deterministic forces," Ann. Phys. (Leipzig) 9, pp. 741-754, 2000.

32. R. Rozenfeld, J. A. Freund, A. Neiman, and L. Schimansky-Geier, "Noise-induced phase synchronization enhanced by dichotomic noise," Phys. Rev. E 64, pp. 051107-1-7, 2001.

33. B. McNamara, K. Wiesenfeld, "Theory of stochastic resonance," Phys. Rev. A 39, pp. 4854-4869, 1989.

34. P. Talkner, "Statistics of entrance times," Physica A 325, pp. 124-135, 2003. 